\title{
Surgery after induction chemo or immunotherapy for locally advanced NSCLC
}

\author{
Filippo Lococo ${ }^{1 \#}$, Carolina Sassorossi ${ }^{1 \#}$, Ciro Mazzarella ${ }^{2}$, Emanuele Vita ${ }^{3}$, Fausto Leoncini ${ }^{4}$, \\ Antonella Martino ${ }^{2}$, Dania Nachira ${ }^{1}$, Marco Chiappetta ${ }^{1}$, Alfredo Cesario ${ }^{1}$, Rocco Trisolini ${ }^{4}$, Emilio Bria ${ }^{3}$, \\ Stefano Margaritora ${ }^{1}$
}

${ }^{1}$ Department of Thoracic Surgery, ${ }^{2}$ Unit of Radiotherapy, ${ }^{3}$ Unit of Oncology, ${ }^{4}$ Unit of Intervention Pneumology, Fondazione Policlinico Universitario “A. Gemelli”, IRCCS, Università Cattolica del Sacro Cuore, Rome, Italy

\#These authors contributed equally to this work.

Correspondence to: Filippo Lococo, MD. Department of Thoracic Surgery, Fondazione Policlinico Universitario "A. Gemelli”, IRCCS, Università Cattolica del Sacro Cuore, L.go F. Vito n 8, 00168 Rome, Italy. Email: filippo_lococo@yahoo.it.

Received: 12 April 2020; Accepted: 14 May 2020; Published: 25 November 2020.

doi: $10.21037 /$ ccts-20-78

View this article at: http://dx.doi.org/10.21037/ccts-20-78

\section{Introduction}

Lung cancer stays as the leading cause of cancer mortality worldwide accounting for 1.8 million newly diagnoses per year (13\% of all cancers diagnosed) (1). Non-small-cell lung cancer (NSCLC) represents more than $80 \%$ of total lung cancer cases of which $19 \%$ are early-stage lung cancers, $25 \%$ locally-advanced and $56 \%$ of patients were diagnosed with distant metastases (2).

Great interest has been placed in the treatment of NSCLC; however, while for early stage lung cancers, survival has increased and guidelines substantially agree on the therapeutic pathway, treatment, for locally-advanced NSCLC, has not been adequately standardized and survival outcomes are almost disappointing, with no remarkable improvements in the last 30 years.

A wide range of treatment options are imaginable for stage III lung cancer. The $8^{\text {th }}$ edition of the American Joint of Committee on Cancer's tumor, node, and metastasis (TNM) staging system classifies locally-advanced NSCLC as IIIA, IIIB, and IIIC (3). While technical advancements increased feasibility of complex resections of locally-invasive tumors, limited evidence exists, supporting extended resections with curative intent for NSCLC-patients with mediastinal nodal disease $(4,5)$. In stage IIIA and IIIB cancer patients, where surgery was the only treatment performed, relapse occurred in more than $80 \%$ of cases, locally in about $1 / 3$ of patients, distantly or combined in about $2 / 3$ of patients. Based on these evidences, multimodal care strategies should be considered.

\section{Locally advanced NSCLC: clinical scenarios and the role of induction therapy}

Locally advanced NSCLC should be classified, if possible, resectable stage III NSCLC and non resectable stage III NSCLC based on radiological and radiometabolic examinations. The NCCN and AIMO guidelines suggest different care strategies for resectable and non resectable stage III NSCLC.

Only in very selective cases an upfront surgery is indicated followed by chemo- and radiotherapy as adjuvant treatments. Surgery can be considered in cases where the mediastinal involved is very limited-single station not bulky N2 disease-or to avoid very harmful and invasive surgical alternatives, like pneumonectomy, after radio-/ chemotherapy.

The alternative is to perform inductive treatment followed by surgical resection in patients considered clinically fit for surgery. This approach is common in highvolume expert tertiary centers despite evidence suggesting neoadjuvant therapy as less effective (as reported in detail below). An analyses of 15 randomised controlled trials (2,385 patients, mainly stage IB-IIIA), showed a significant survival benefit performing preoperative chemotherapy resulting in a $13 \%$ reduced relative risk of death (6). 


\section{Role and modalities of induction therapy protocols}

From a theoretical point of view, induction treatment in locally-advanced NSCLC should have the following purposes:

(I) To increase resectability by downstaging lung cancers;

(II) To reduce the resection extent;

(III) To reduce the local and distance recurrence rate controlling microscopic distant metastasis;

(IV) To increase overall survival.

However, it should be considered that:

(I) Surgery needs to be postponed for 2 to 4 weeks to allow recovery from induction therapy;

(II) Surgery may be further delayed because recovery takes longer than 2 to 4 weeks;

(III) During recovery time progression of disease might occur, if induction therapy was ineffective;

(IV) Surgery after induction therapy seems to correlate with a high-risk post-operative course.

\section{Locally advanced NSCLC: clinical scenarios}

Since locally-advanced NSCLC is a very heterogeneous disease, treatment approaches depend on tumor stage and TNM indicators.

\section{Locally-advanced resectable NSCLC}

As reported above, the treatment of resectable LA-NSCLC is debatable (1). The decision of surgical resectability is crucial and is usually based on radiological examination (or radiometabolic results) taking neoplastic contact/infiltration with vascular structures and neighbour organs into account.

When locally-advanced NSCLC is considered resectable, upfront surgery could be considered a feasible option [followed by adjuvant chemo(radio)therapy] in selected cases, like:

(I) T3/4-N0 NSCLC where lymph nodal disease had been excluded by invasive methods;

(II) T1-3/N2 NSCLC in presence of single station $\mathrm{N} 2$-disease (excluding station 7) where other mediastinal lymph nodal stations have been biopsied and proved to be benign (7);

(III) T1-3/N2 NSCLC where surgical excision of the tumor is achievable only performing a pneumonectomy; in such cases, induction therapy followed by pneumonectomy in N2 disease could be a very questionable (and harmful) procedure and some authors (6) suggest to perform upfront surgery followed by adjuvant chemotherapy.

In the remaining cases, when a multimodal approach is planned, the decision to perform pre-operative chemotherapy (eventually combined with radiotherapy) is preferable, as showed in a systematic review and metaanalysis of the NSCLC meta-analysis collaborative group (6). Indeed, induction chemotherapy remarkably improves overall long-term survival and recurrence-free survival in resectable NSCLC, suggesting it as a valid and feasible treatment option for most of them.

As well, the final decision to proceed with resection after induction chemotherapy must include a detailed pre-op pulmonary function evaluation and an accurate feasibility assessment for radical resection during surgery (8).

\section{NO and N1-resectable disease}

In some Stage-III NSCLC-patients with N0-N1 disease, surgery is typically the initial therapy (5). Generally, the treatment approach of stage IIIA patients without mediastinal nodal involvement is similar to stage II patients. When the tumor is node-negative (N0), the most significant factor influencing the prognosis is local control. Our team compared long-term results in T3T4/N0 NSCLC-patients who received induction treatment prior to surgery and patients who directly underwent surgery (9). No significant differences were found comparing long-term survival of the induction treatment group and the upfront surgery group, but it emerged that patients who underwent an incomplete (R1/R2) tumor resection the mortality rate is 5 times higher compared with R0 patients. It was concluded that surgical resection following an inductive treatment is a safe and reasonable treatment in T3-T4/N0 NSCLC-patients, and an improved survival could be expected in patients undergone radical resection and complete pathologic response (9).

\section{N2-resectable disease}

Although definitive chemo-radiation remains the standard of care for cN2-NSCLC, alternative approaches such as induction chemo(radio)therapy followed by surgical resection may be considered for a selected subset of patients. Expert opinions are a major driver of clinical decision making when evidence is substantially lacking. A survey of 513 thoracic surgeons showed that $96 \%$ of surgeons would recommend surgery as part of multi-modality strategy 
of care in NSCLC-patient with single-station not-bulky N2-disease (4). Unlikely, when confronted with NSCLC presenting with bulky multi-station N2-disease, $82 \%$ continued to recommend surgery as part of the therapy. However, when surgical resection may be performed after an induction treatment the early and long-term results may be optimal with low post-op risks and a good chance of complete surgical resection.

\section{Locally-advanced non resectable NSCLC}

"Unresectable" disease defines a neoplasm that, even after induction therapy, is deemed to be not radically resectable (R0) based on evaluation within a multidisciplinary team, including an experienced thoracic surgeon. Chemoradiotherapy is recommended in patients with unresectable stage IIIA and IIIB disease (10).

Sequential chemo-radiotherapy (induction chemotherapy followed by radiotherapy), usually administered at a dose of 60-66 Gy in 30-33 fractions over 6-7 weeks, has been compared to concurrent chemo-radiotherapy in several phase-III trials and in a meta-analysis (11). A concurrent approach is considered the best therapeutic option for fit patients, resulting in improved 5-year survival rates because of improved loco-regional control. The decrease of locoregional failure observed with concomitant combinations might be related to the radiosensitizing effect of cisplatinbased chemotherapy.

An increase of acute esophageal toxicity in the concomitant treatment arm compared with sequential treatment arm has been demonstrated and the intensification of both radiotherapy and concurrent chemotherapy resulted in an excessive toxicity or incomplete treatment $(11,12)$. However, the sequential approach can be considered the treatment of choice for elderly and/or unfit patients $(13,14)$.

\section{Neoadjuvant protocols: chemotherapy vs. chemoradiotherapy}

Several meta-analyses about neoadjuvant chemotherapy in NSCLC showed a remarkable survival advantage over surgery alone [hazard ratio (HR): 0.8; 5-year survival advantage: $5 \%$ ] $(13,14)$. No consensus was achieved whether a "fixed number" of preoperative chemotherapy cycles should be administered in the absence of dose-limiting toxicities. The current recommendation is to use the same regimen employed in the adjuvant setting, generally four cycles. Platinum-based chemotherapy is an essential part of the treatment of locally-advanced NSCLC as it improves survival in resectable as well unresectable tumors.

The main schemes used are cisplatin-etoposide or carboplatin-paclitaxel regimens, but recently, novel chemotherapy agents (vinorelbine, gemcitabine, irinotecan, docetaxel, pemetrexed, and S-1 are increasingly administered concomitant with thoracic radiation for locally advanced NSCLC showing efficacy in clinical trials. However, there are only few studies that directly compared these new regimens with conventional options, and accordingly, the optimal chemotherapy regimen concurrently used with thoracic radiation is still debated (15).

The aim of adding radiotherapy before surgery is to improve overall survival by decreasing local tumor relapse. In particular, reducing local recurrences could be the primary endpoint where local failure impacts on the overall quality of life, such as for Pancoast syndrome (16).

In potentially resectable NSCLCs invading the superior sulcus, an induction concurrent chemo-radiotherapy followed by surgical resection may be considered the standard of care. Basically, the same strategy could be applied for potentially-resectable T3-T4 central NSCLC in highly selected cases and experienced centres.

The recommendation to add chemotherapy to radiotherapy is based on studies showing an improved overall long-term outcome for this approach compared with induction radiotherapy alone, with a meta-analysis demonstrating an absolute benefit of $2.2 \%$ at 5 years (HR: 0.89; 95\% CI: 0.81-0.98; $\mathrm{P}=0.02$ ) (11).

Thus, considering these aspects, one of the future challenges is to tailor combination treatments, taking into account both tumor and toxicity profiles.

\section{Surgery after neoadjuvant treatment: criticism and results}

Selection of the optimal candidates for surgery remains a crucial point in daily clinical practice and is strongly influenced by current restaging capabilities.

As reported in the IASLC Consensus Report [2003], NSCLC-patients who underwent complete surgical resection after a complete or partial response to induction therapy is associated with improved long-term survival. Therefore, restaging after induction therapy has gained an increasing interest in the scientific community. Our team showed that a pathological complete response could be achieved in a significant proportion of cases after induction treatment (27\% of patients underwent surgical resection) 
with a statistically improve 5 -year long-term survival (LTS) of $64 \%$ (17).

The appropriate timing of surgical intervention after inductive treatments continues to remain an unsolved topic. Rice et al. (18) showed a significant drop in survival probability in patients with delayed intervention. Randomized trials study the use of induction radiochemotherapy in locally advanced NCSLC minimizing the interval from completion of induction radio-chemotherapy (usually the final dose of radiotherapy) to surgery, which could potentially reduce morbidity associated with surgery in patients with fibrosis or pneumonitis.

Swaminath et al. (19) observed, in the post hoc analysis of the Intergroup-Trial, decreased treatment-related deaths in patients who underwent lobectomy than in those who underwent pneumonectomy, after chemo-radiation suggesting that lobectomy should be always preferred (when technically feasible) after chemo-radiation in patients with resectable stage III NSCLC.

In resectable locally-advanced NSCLC, chemotherapy demonstrated to be superior in terms of survival, before surgery, rather than after (20) improving statistically significant median survival of 26 vs. 8 months $(\mathrm{P}<0.001)$ for those randomized to chemotherapy before surgery compared to those randomized to surgery first. Notably, best evidence supporting the neoadjuvant approach in resectable patients with NSCLC is based on a phase II rather than phase III studies. The most critical and still unsolved question is to identify those individuals who most likely benefit from either adjuvant or neoadjuvant chemotherapy (21).

\section{Neoadjuvant immunotherapy}

Despite, major therapeutic successes-with increased overall survival-has been achieved, in recent years, by targeted therapy and immunotherapy in advanced-stage NSCLCs, remains surgery the first option for patients with resectable NSCLC. Efforts are focused on replication of the success achieved using innovative treatments in advanced NSCLC stages and especially in early/locally-advanced stages as induction treatments (22). The idea of cancer immunotherapy originated with a deeper understanding of the immune system, which can recognize malignant cells inducing immune responses to eliminate cancer cells (23).

The relative efficacy of programmed death 1 (PD-1) and/or programmed death ligand 1 (PD-L1) antibodies in metastatic NSCLCs expanded their clinical use to other stages. The complex and diverse nature of stage-III tumors invites to incorporate immunotherapy into treatment protocols in both induction and consolidation settings (24). The relative success of this strategy, compared to induction platinum-based chemotherapy, will hopefully be clarified by the currently accruing clinical trials (25).

\section{Immunotherapy in NCCN guidelines (version 1.2020)}

According to latest guidelines, immunotherapy (durvalumab) is now indicated in some specific subsets of NSCLC cases, for example for unresectable NSCLC stage IIIA (T4-N0/1) as initial treatment or following inductive chemotherapy. Other indications are unresectable NSCLC stage IIIB (T3-N2) and stage IIIC (T4-N3), usually after concomitant chemo-radiotherapy. For NSCLC stage IV, the indication mostly depends on the percentage of PD-L1 expression and on histological diagnosis (adenocarcinoma $v s$. squamous cell lung cancer) (2).

\section{Neoadjuvant PD-1 blockade}

Antibodies that block PD-1 protein improve long-term outcome in advanced-stages NSCLC-patients, but their role in resectable NSCLC has not been clarified yet.

PD-1 pathway blockade may have enhanced antitumor effects in patients with early-stage lung cancer owing to greater fitness of host immunity and reduced tumor clonal heterogeneity (21). Induction immunotherapy is an attractive treatment approach, considering that the primary tumor may be leveraged as an antigen source for expansion/activation of tumor-specific T-cells as well as systemic control of micrometastases. In addition, induction approaches provide an opportunity to analyse the in vivo effect of PD-1 blockade on the primary-tumor microenvironment and peripheral blood (26).

The ongoing phase II NEOMUN trial investigates the role of pembrolizumab in resectable N+ Stage IIIIIA NSCLC. In particular, the NEOMUN trial is one of the first clinical trials exploring, as primary endpoint, the feasibility of a multimodal approach including induction immunotherapy with pembrolizumab as an investigational drug (27). Moreover, the efficacy of an anti-PD1-treatment on clinical and pathologic tumor response will be evaluate. Secondary end-point is to evaluate the impact of induction immunotherapy on both overall and disease-free survivals. Pembrolizumab will be administrated at a fixed dose of $200 \mathrm{mg}$ every 3 weeks for 2 cycles (day- 0 and 21). Then 
surgical resection with curative intent will be performed in all patients who met the eligibility criteria.

\section{Work in progress: PD-L1 expression enbanced by cisplatinum}

Fournel et al. demonstrated (in vivo) that cisplatinbased induction treatment significantly increases PD-L1 staining in both tumor and immune cells from a tumoral microenvironment.

The results provide a solid and reliable basis for further explorations, reinforcing the rationale for several ongoing clinical trials, which test combination therapies including PD-L1 and cytotoxic drugs like cisplatin. This raises the question how relevant sequential re-evaluation of PD-L1 status performance is to select the ideal subset of patients (28).

\section{Conclusions}

Future treatments, for locally advanced NSCLC, should better control local and microscopic systemic disease, because the actual 5-year survival of stage-III NSCLC-patients undergone surgical resection only is still disappointing. One possibility to improve surgical outcomes of tumors considered technically resectable is the administration of inductive therapy before surgery. In the last two decades, several studies investigated optimal induction chemo, radio or (more recently) immunotherapy regimens that can be combined with surgical treatment. Based on the results of those clinical studies, multimodality therapy is considered to be an appropriate treatment approach for stage IIIA NSCLC patients. However, optimal treatment strategies are still evolving and satisfying survival results are far from being achieved.

\section{Acknowledgments}

Funding: None.

\section{Footnote}

Provenance and Peer Review: This article was commissioned by the Guest Editors (Davide Tosi and Alessandro Palleschi) for the series "The Treatment of Locally Advanced Lung Cancer" published in Current Challenges in Thoracic Surgery. The article has undergone external peer review.

Conflicts of Interest: All authors have completed the ICMJE uniform disclosure form (available at https://ccts.amegroups. com/article/view/10.21037/ccts-20-78/coif). The series "The Treatment of Locally Advanced Lung Cancer" was commissioned by the editorial office without any funding or sponsorship. MC serves as an unpaid editorial board member of Current Challenges in Thoracic Surgery from December 2019 to November 2021. The authors have no other conflicts of interest to declare.

Ethical Statement: The authors are accountable for all aspects of the work in ensuring that questions related to the accuracy or integrity of any part of the work are appropriately investigated and resolved.

Open Access Statement: This is an Open Access article distributed in accordance with the Creative Commons Attribution-NonCommercial-NoDerivs 4.0 International License (CC BY-NC-ND 4.0), which permits the noncommercial replication and distribution of the article with the strict proviso that no changes or edits are made and the original work is properly cited (including links to both the formal publication through the relevant DOI and the license). See: https://creativecommons.org/licenses/by-nc-nd/4.0/.

\section{References}

1. Vansteenkiste J, Crinò L, Dooms C, et al. 2nd ESMO Consensus Conference on Lung Cancer: early-stage nonsmall-cell lung cancer consensus on diagnosis, treatment and follow-up. Ann Oncol 2014;25:1462-74.

2. Ettinger DS, Wood DE, Aggarwal C, et al. NCCN guidelines insights: non-small cell lung cancer, version 1.2020. J Natl Compr Canc Netw 2019;17:1464-72.

3. Gospodarowicz MK, Brierley JD, Wittekind C. TNM classification of malignant tumours. Hoboken: Wiley, 2017.

4. Veeramachaneni NK, Feins RH, Stephenson BJ. Management of stage IIIA non-small cell lung cancer by thoracic surgeons in North America. Ann Thorac Surg 2012;94:922-6; discussion 926-8.

5. Bryan DS, Donington JS. The role of surgery in management of locally advanced non-small cell lung cancer. Curr Treat Options Oncol 2019;20:27.

6. NSCLC Meta-analysis Collaborative Group. Preoperative chemotherapy for non-small-cell lung cancer: a systematic review and meta-analysis of individual participant data. Lancet 2014;383:1561-71.

7. Tsitsias T, Boulemden A, Ang K, et al. The N2 paradox: similar outcomes of pre- and postoperatively identified 
single-zone N2a positive non-small-cell lung cancer. Eur J Cardiothorac Surg 2014;45:882-7.

8. Watanabe SI, Nakagawa K, Suzuki K, et al. Neoadjuvant and adjuvant therapy for stage III non-small cell lung cancer. Jpn J Clin Oncol 2017;47:1112-8.

9. Lococo F, Cesario A, Margaritora S, et al. Induction therapy followed by surgery for T3-T4/N0 non-small cell lung cancer: long-term results. Ann Thorac Surg 2012;93:1633-40.

10. Park K, Vansteenkiste J, Lee KH, et al. Pan-Asian adapted ESMO Clinical Practice Guidelines for the management of patients with locally-advanced unresectable non-smallcell lung cancer: a KSMO-ESMO initiative endorsed by CSCO, ISMPO, JSMO, MOS, SSO and TOS. Ann Oncol 2020;31:191-201.

11. Aupérin A, Le Péchoux C, Rolland E, et al. Meta-analysis of concomitant versus sequential radiochemotherapy in locally advanced non-small-cell lung cancer. J Clin Oncol 2010;28:2181-90.

12. Liu T, He Z, Dang J, et al. Comparative efficacy and safety for different chemotherapy regimens used concurrently with thoracic radiation for locally advanced non-small cell lung cancer: a systematic review and network metaanalysis. Radiat Oncol 2019;14:55.

13. Brunelli A, Charloux A, Bolliger CT, et al. ERS/ESTS clinical guidelines on fitness for radical therapy in lung cancer patients (surgery and chemo-radiotherapy). Eur Respir J 2009;34:17-41.

14. Cheema PK, Rothenstein J, Melosky B, et al. Perspectives on treatment advances for stage III locally advanced unresectable non-small-cell lung cancer. Current Oncology 2019;26:37-42.

15. Burdett S, Stewart L, Auperin A, et al. Chemotherapy in non-small-cell lung cancer: an update of an individual patient data meta-analysis. J Clin Oncol 2005;23:924-5; author reply 925-6.

16. Blumenthal GM, Bunn PA Jr, Chaft JE, et al. Current status and future perspectives on neoadjuvant therapy in lung cancer. J Thorac Oncol 2018;13:1818-31.

17. Lococo F, Cesario A, Margaritora S, et al. Long-term results in patients with pathological complete response after induction radiochemotherapy followed by surgery for locally advanced non-small-cell lung cancer. Eur J Cardiothorac Surg 2013;43:e71-81.

18. Rice JD, Heidel J, Trivedi JR, et al. Optimal surgical timing after neoadjuvant therapy for stage IIIa non-small cell lung cancer. Ann Thorac Surg 2020;109:842-7.

19. Swaminath A, Vella ET, Ramchandar K, et al. Surgery after chemoradiotherapy in patients with stage III (N2 or N3, excluding T4) non-small-cell lung cancer: a systematic review. Current Oncology 2019;26:e398-404.

20. Rosell R, Gomez-Codina J, Camps C, et al. A randomized trial comparing preoperative chemotherapy plus surgery with surgery alone in patients with non-small cell lung cancer. N Engl J Med 1994;330:153-8.

21. Strauss GM. Adjuvant vs neoadjuvant chemotherapy in resectable NSCLC: is that the real question? Oncology (Williston Park) 2009;23:534-6, 538.

22. Zhu J, Li R, Tiselius E, et al. Immunotherapy (excluding checkpoint inhibitors) for stage I to III non small cell lung cancer treated with surgery or radiotherapy with curative intent. Cochrane Database Syst Rev 2017;12:CD011300.

23. Bertolaccini L, Casiraghi M, Spaggiari L. Immunotherapy in the neoadjuvant settings: a new challenge for the thoracic surgeon? Interact Cardiovasc Thorac Surg 2020;30:1-3.

24. Bott MJ, Yang SC, Park BJ, et al. Initial results of pulmonary resection after neoadjuvant nivolumab in patients with resectable non-small cell lung cancer. J Thorac Cardiovasc Surg 2019;158:269-76.

25. Yeh J, Marrone KA, Forde PM, et al. Neoadjuvant and consolidation immuno-oncology therapy in stage III nonsmall cell lung cancer. J Thorac Dis 2018;10:S451-9.

26. Forde PM, Chaft JE, Smith KN, et al. Neoadjuvant PD-1 blockade in resectable lung cancer. $\mathrm{N}$ Engl J Med 2018;378:1976-86.

27. Eichhorn F, Klotz LV, Bischoff H, et al. Neoadjuvant antiprogrammed death-1 immunotherapy by Pembrolizumab in resectable nodal positive stage II/IIIa nonsmall-cell lung cancer (NSCLC): the NEOMUN trial. BMC Cancer 2019;19:413.

28. Fournel L, Wu Z, Stadler N. Cisplatin increases PD-L1 expression and optimizes immune check-point blockade in non-small cell lung cancer. Cancer Lett 2019;464:5-14.

doi: $10.21037 /$ ccts-20-78

Cite this article as: Lococo F, Sassorossi C, Mazzarella C, Vita E, Leoncini F, Martino A, Nachira D, Chiappetta M, Cesario A, Trisolini R, Bria E, Margaritora S. Surgery after induction chemo or immunotherapy for locally advanced NSCLC. Curr Chall Thorac Surg 2020;2:42. 